\title{
Enhanced photoelectrocatalysis using a bimetallic plasmonic near-perfect absorber
}

Qi Xiao,,$^{1,+}$ Calum Kinnear, ${ }^{1,+}$ Timothy U. Connell, ${ }^{2,+}$ Muhammad Kalim Kashif, ${ }^{3}$ Christopher D. Easton, ${ }^{1}$ Aaron Seeber, ${ }^{1}$ Laure Bourgeois, ${ }^{4}$ Gus. O. Bonin, ${ }^{2}$ Noel W. Duffy, ${ }^{3}$ Anthony S. R. Chesman, ${ }^{*}, 1,5$ and Daniel E. Gómez ${ }^{*}, 2$

${ }^{1}$ CSIRO Manufacturing, Bayview Ave, Clayton, VIC 3168, Australia

${ }^{2}$ RMIT University, Melbourne, VIC 3000, Australia

${ }^{3}$ CSIRO Energy, Bayview Ave, Clayton, VIC 3168, Australia

${ }^{4}$ Monash Centre for Electron Microscopy and Department of Materials Science and Engineering, Monash University, Clayton, VIC 3800, Australia

${ }^{5}$ Melbourne Centre for Nanofabrication, Australian National Fabrication Facility, Clayton, VIC 3168, Australia

${ }^{+}$These authors contributed equally to the work.

\section{ABSTRACT}

Plasmonic noble metal nanoparticles exhibit intense interactions with light but are inherently chemically unreactive. Alloying gold with catalytic palladium forms a hybrid nanostructured catalyst incorporating each metal's benefits. In this work we demonstrate a robust method for preparing alloyed nanoparticles without the need for colloidal synthesis or time-consuming lithography. This method results in uniform, densely packed monolayers of AuPd alloyed nanoparticles suitable for inclusion in electromagnetic perfect absorbers that further strengthen existing light-matter interactions. These materials demonstrate a strong photocathodic response not possible using monometallic Au nanoparticles on a n-type spacer and exhibit higher photon-to-energy efficiencies than Pd analogues. The alloying of plasmonic $\mathrm{Au}$ and catalytic $\mathrm{Pd}$ and subsequent incorporation in a highly absorbing material represents a promising step towards efficient photoelectrocatalysts that operate under real-world conditions.

KEYWORDS: AuPd alloy, photoelectrocatalysis, plasmons, near perfect absorber, physical vapour deposition 


\section{Introduction}

Photoelectrochemical water splitting promises the storage of solar energy as high-energy hydrogen fuel, while also a green alternative to steam reforming for hydrogen production. A successful photocatalyst must both strongly absorb light and demonstrate a high reaction efficiency. Plasmonic noble metal nanoparticles are effective visible light harvesters that generate energetic electrons through the excitation of localised surface plasmon resonances. ${ }^{1-6}$ When coupled with a suitable semiconductor, such as $\mathrm{TiO}_{2}$, these hot electrons are extracted across a Schottky junction, leaving an energetic hole that affords water oxidation at the nanoparticle surface. ${ }^{7-8}$ The efficiency of electron extraction across the Schottky junction is theoretically defined by the work function of both the metal and the semiconductor, however the properties of the metal-semiconductor interface also play a decisive role..$^{9-10}$ Charge-transfer is further impeded by competitive electron relaxation pathways ${ }^{11}$ and there remains a significant need for highly photon-to-hydrogen efficient plasmonic photoelectrochemical systems. ${ }^{12-13}$

Noble metals that yield optically active plasmonic nanoparticles, i.e. Au and $\mathrm{Ag}$, typically exhibit poor reactivity compared with transition metals common in catalysis, like Pd or Pt. Combining the best properties of each is possible using bimetallic heterostructures where a plasmonic antenna absorbs and transfers energy to the catalytic reactor. ${ }^{14-17}$ Alloyed nanoparticles can achieve similar synergistic results in a single particle, without the difficult preparation often necessary for fused bimetallic systems. ${ }^{18}$ Unlike fused systems, the electronic states of alloyed atoms is affected by the difference in electronegativity of the constituent elements, changing the electron density of their respective valence bands. ${ }^{19-21}$ These differences can change the enthalpy of reactant adsorption and chargetransfer within alloyed nanostructures leading to enhanced reaction rates. ${ }^{22-24}$ Finally, alloying can suppress light-induced interband excitations of Au and instead favour intraband excitations that result in higher hot-electron energies and injection efficiencies. ${ }^{25}$

Further improvements to light absorption and charge-extraction are possible when nanoparticles are incorporated in electromagnetic perfect absorbers. ${ }^{26-27}$ Our development of plasmonic near-perfect absorbers, which strongly absorb incident light and localize this energy at the nanoparticle surface, promises photocatalysts that maintain high activity whilst minimizing the cost of precious metal components..$^{28-31}$ These materials, typically fabricated via vapour deposition, comprise of three thin layers: a metallic mirror, an optically transparent spacer and the absorbing nanostructured layer. Alloyed nanoparticles are rarely used in such solid-state devices due to their wet-chemical synthesis. ${ }^{32-}$

${ }^{33}$ Integrating colloidal nanoparticles onto a thin film as a self-assembled monolayer is often difficult, particularly with the high-surface coverage often desired in catalysis. ${ }^{34}$ Similarly, time-intensive nanolithography is typically required when alloyed nanostructures are fabricated using evaporative techniques..$^{35-40}$ As such, easy to fabricate alloy nanoparticles with well-defined size, shape and composition remain an attractive target in heterogenous photocatalytic design.

Here we present the fabrication of a plasmonic near-perfect absorber with a dense, uniform monolayer of AuPd alloyed nanoparticles as the absorbing layer. The nanoparticles were prepared by dewetting thin films $(<4 \mathrm{~nm})$ of each metal on a dielectric surface and extensive characterisation confirmed the lack of other bimetallic phases (i.e. antenna-reactor or core-shell structures). Plasmonic near-perfect absorbers containing an alloyed nanoparticle layer demonstrated both strong onresonance absorption of incident light (up to 97\%) and an improved photocatalytic response, compared to either monometallic derivative, for the photoelectrochemical reduction of water. 


\section{Results and Discussion}

\section{Fabrication of alloyed near perfect absorbers}

The fabrication of an alloyed plasmonic near-perfect absorber using an entirely physical vapour deposition-based approach extends on our previous work with monometallic analogues. ${ }^{28-30}$ This architecture comprised a metallic mirror and absorbing layer separated by a high bandgap semiconductor (Figure 1A). Consecutive electron beam evaporation of $\mathrm{Au}(100 \mathrm{~nm})$ and $\mathrm{TiO}_{2}(30 \mathrm{~nm})$ thin films onto glass substrates gave the mirror and semiconductor spacer layer, respectively. Spacer thickness was carefully selected to ensure strong coupling between the localised surface plasmon of the nanoparticles and the Fabry-Pérot cavity mode. ${ }^{27}$ The absorbing layer consisted of alloyed AuPd nanoparticles, fabricated by physical vapour deposition of thin $\mathrm{Au}$ and Pd films (1-3 $\mathrm{nm}$ each) prior to annealing in a tube furnace under inert atmosphere ( $\left.\mathrm{Ar}, 500{ }^{\circ} \mathrm{C}, 30 \mathrm{~min}\right)$. Annealing in air formed significant amounts of PdO, as identified by X-ray diffraction (XRD, see Supporting Information Figure S1). Inductively coupled plasma mass spectrometry (ICP-MS) confirmed the accuracy of thin film deposition; alloyed nanoparticles fabricated on a Si wafer using $2 \mathrm{~nm}$ films of each metal exhibited equimolar amounts of $\mathrm{Au}$ and $\mathrm{Pd}\left(19 \mathrm{nmol} \mathrm{\textrm {cm } ^ { - 2 }}\right)$ after digestion and analysis (Table 1). X-ray photoelectron spectroscopy (XPS) indicated the formation of a Schottky barrier; characterized by a slight positive shift in Ti $2 p_{3 / 2}$ binding energy, regardless of alloy composition, resulting from electron donation to the absorbing nanoparticles (Table 1).

Scanning electron microscopy (SEM) of the plasmonic near perfect absorber before annealing revealed a continuous coarse surface that coalesced into homogeneous, uniformly distributed nanoparticles once annealed (Figure 1B, C). We found it crucial to initially deposit Au due to greater wetting of $\mathrm{Pd}$ on the $\mathrm{TiO}_{2}$ surface; direct deposition of $\mathrm{Pd}$ onto the semiconductor caused significant ripening during annealing and discreet nanoparticles did not form (Figure S2). Grazing incidence XRD further supported the formation of AuPd alloy nanoparticles (Figure 1D). The measured lattice constant $\left(3.97 \AA\right.$ ) indicated a homogeneous alloy across the $\mathrm{TiO}_{2}$ surface, intermediate between $\mathrm{Au}$ $(4.08 \AA)$ and $\mathrm{Pd}(3.89 \AA)$. We also observed both nanocrystalline anatase $\mathrm{TiO}_{2}$ of the semiconductor spacer along with a pure Au phase, which we assigned to the metallic mirror, at high grazing angles $\left(\omega>0.35^{\circ}\right)$.

(A)
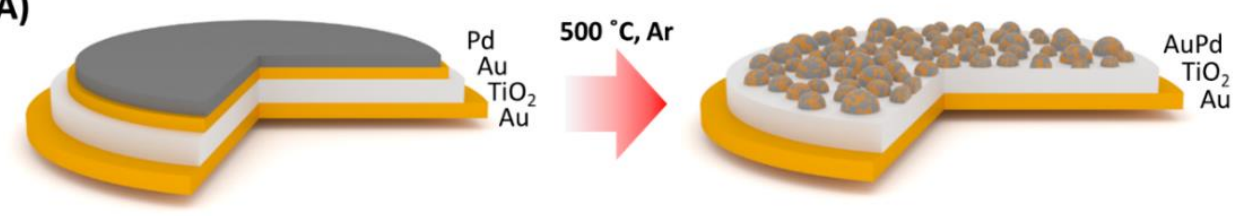

(B)

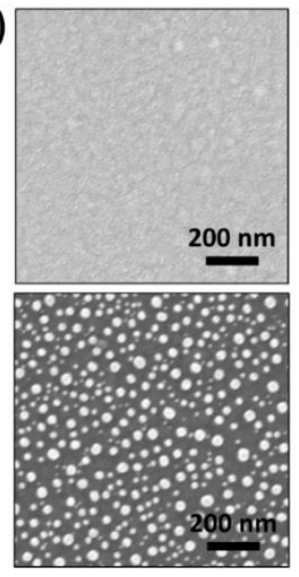

(C)

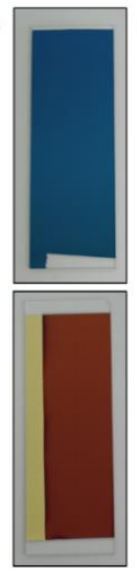

(D)

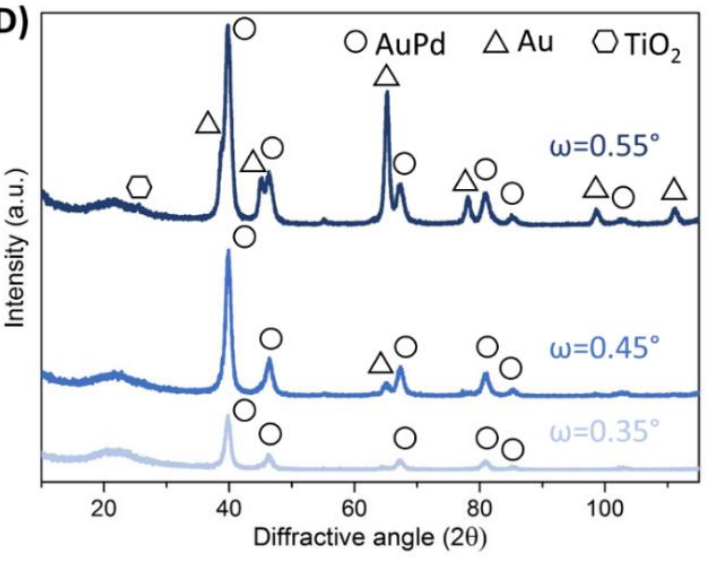

Figure 1. (a) Plasmonic near-perfect absorbers were fabricated via thin film vapour deposition and annealing under inert atmosphere. (b) Scanning electron microscopy of surface before (top) and after annealing (bottom) along with (c) photographs of entire glass slide substrate. (d) Grazing incidence X-ray diffraction of fabricated plasmonic near-perfect absorbers. 
Scanning transmission electron microscopy (STEM) revealed spheroid nanoparticles $10-15 \mathrm{~nm}$ in size, with a projected aspect ratio between 1-2 (Figure 2A, Figure S3). Elemental mapping of approximately 100 nanoparticles using energy-dispersive $X$-ray spectroscopy found an even distribution ( $\pm 5 \%$ ) of $\mathrm{Au}$ and $\mathrm{Pd}$ both within and between individual nanoparticles without any segregation (additional images Figure S4). The consistent contrast across hundreds of nanoparticles using high-angle annular dark field (HAADF-)STEM measurements (Figure 2B) also supported alloy formation. This technique is sensitive to atomic number ( $Z$, where higher $Z$ values exhibit greater signal intensity), yet we saw no evidence over the many hundred nanoparticles imaged of the high contrast expected from either pure $\mathrm{Au}(\mathrm{Z}=79)$ or $\mathrm{Pd}(\mathrm{Z}=46)$ nanoparticles. High-resolution STEM images revealed numerous defects and twin boundaries within the crystalline nanoparticles (Figure $\mathbf{2 C}$ ) however, the lattice spacings measured from selected area electron diffraction patterns obtained from hundreds of nanoparticles revealed only a single AuPd face-centred cubic phase. No evidence of split rings, resulting from the different lattice parameters of phase separated crystalline Au and Pd, was observed (Figure 2D).
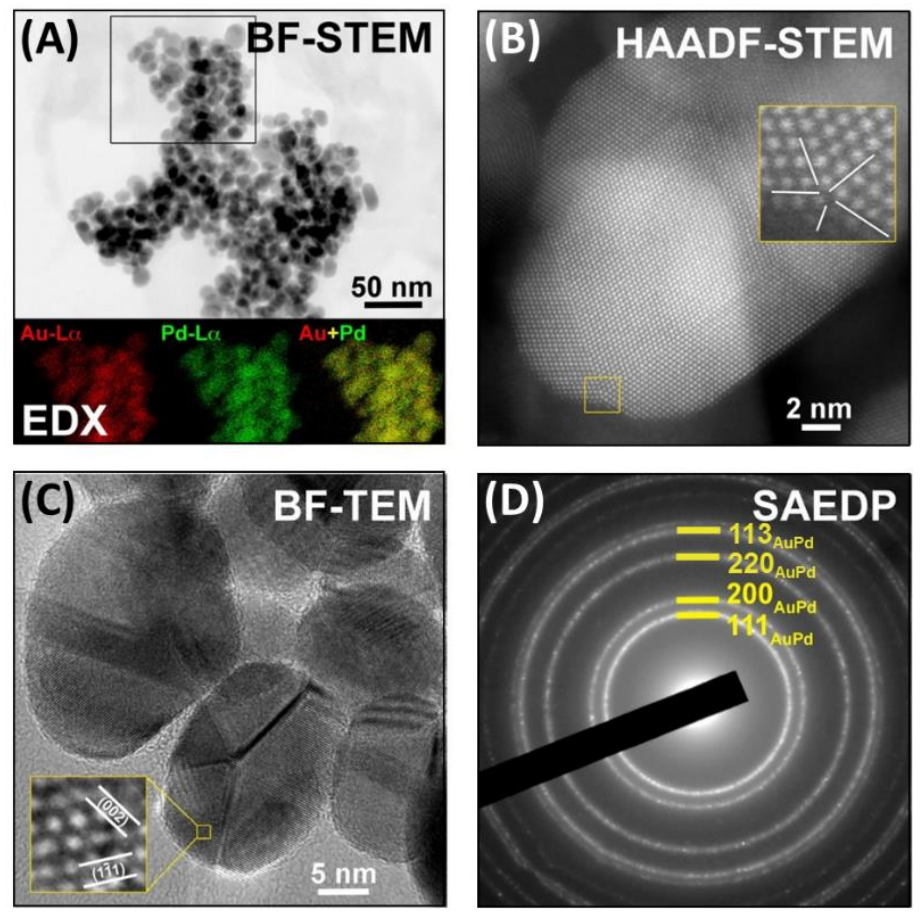

Figure 2. Scanning transmission electron microscopy (STEM) of alloyed AuPd nanoparticles: (a) Bright-field image, with inset showing the elemental mapping using energy-dispersive X-ray (EDX) spectroscopy of area framed in black; (b) High-angle annular dark field (HAADF) image with inset showing a magnified view of the area framed in yellow. White lines indicate twin planes; (c) High resolution bright-field image with inset showing a magnified view of the area framed in yellow. The (002) and (111) lattice planes are clearly visible; (d) Selected-area electron diffraction pattern of a group of nanoparticles, rings indexed according to the AuPd crystal structure.

The atomic ratio of alloyed nanoparticles calculated using X-ray spectroscopy slightly differed to those obtained via ICP-MS (Table 1). We found that surface-sensitive XPS consistently detected a reduced Au content, although grazing incidence measurements did shift closer to the expected value for AuPd ratios of 1:3 and 2:2. As deposited film thicknesses are below the percolation limit of each metal, we speculate that this discrepancy arises from small amounts of Pd that deposit directly on to the dielectric surface. The better wetting of $\mathrm{Pd}$ on $\mathrm{TiO}_{2}$, when compared with $\mathrm{Au}$, likely results in residual $\mathrm{Pd}$ not coalescing into the final alloyed nanoparticles, thereby lowering the measured molar ratio. Contrary to XPS quantification, atomic ratios calculated from extended X-ray absorption fine structure (EXAFS) spectroscopy were consistently higher in Au content, although the associated error of this technique is around 15\%. Additionally, artefacts that arise during EXAFS data analysis often result in discrepant results when compared to elemental compositions measured using more direct spectroscopies. ${ }^{41}$ Finally, we emphasise that the small surface-to-volume ratio of these fabricated 
AuPd nanoparticles (10-15 nm), compared with most oxide-supported metallic catalysts (typically $<5 \mathrm{~nm}$ ), renders EXAFS analysis unsuitable for elucidating increased amounts of one element at the nanoparticle surface. ${ }^{41-42}$

\section{Electronic Properties of Alloyed Nanoparticles}

We next investigated the electronic properties and coordination environment of metal atoms within the alloyed nanoparticles. Photoelectron emission from Au $4 f$ (Figure S5, for fitting of XPS spectra see Figure S6) shifted to lower binding energies upon alloying due to net electron transfer from the less electronegative $\mathrm{Pd}$. We observed a concordant shift in work function using photoelectron spectroscopy in air; alloying with Pd slightly decreased the energy required $(5.03 \mathrm{eV})$ to remove an electron compared to the monometallic Au plasmonic near-perfect absorber (5.10 eV, Figure S7). Increasing Au from $25-75 \%$ exhibited a concomitant decrease in the resulting shift of the XPS $4 f_{7 / 2}$ peak from -0.5 to $-0.2 \mathrm{eV}$, respectively.

The XPS spectrum in the binding energy region of 330-360 eV corresponds to spin-orbit splitting for both Pd $3 d$ (split into $3 d_{3 / 2}$ and $\left.3 d_{5 / 2}\right)$ and Au $4 d\left(4 d_{3 / 2}\right.$ and $\left.4 d_{5 / 2}\right)$. While the Au band exists in a single metallic state, the $\mathrm{Pd} 3 d$ contributions were peak fitted with four distinct doublets using a fixed peak separation of $5.3 \mathrm{eV}$ and an additional singlet representing $\mathrm{Pd}(0)$ plasmon loss around $346.6 \mathrm{eV}$ (Figure $3 \mathrm{~A}-\mathrm{C}$ ). ${ }^{43}$ Both the $\mathrm{Pd}(0) 3 d_{5 / 2}$ peak (labelled as $\mathrm{Pd} 1$, around $334.8 \mathrm{eV}$ ), and $\mathrm{Au} 4 f_{7 / 2}$ peaks shift to lower binding energies in a 1:1 alloy ( -0.4 and $-0.7 \mathrm{eV}$, respectively) and at least $35 \%$ of all $\mathrm{Pd}$ is found in this $\operatorname{Pd}(0)$ state.
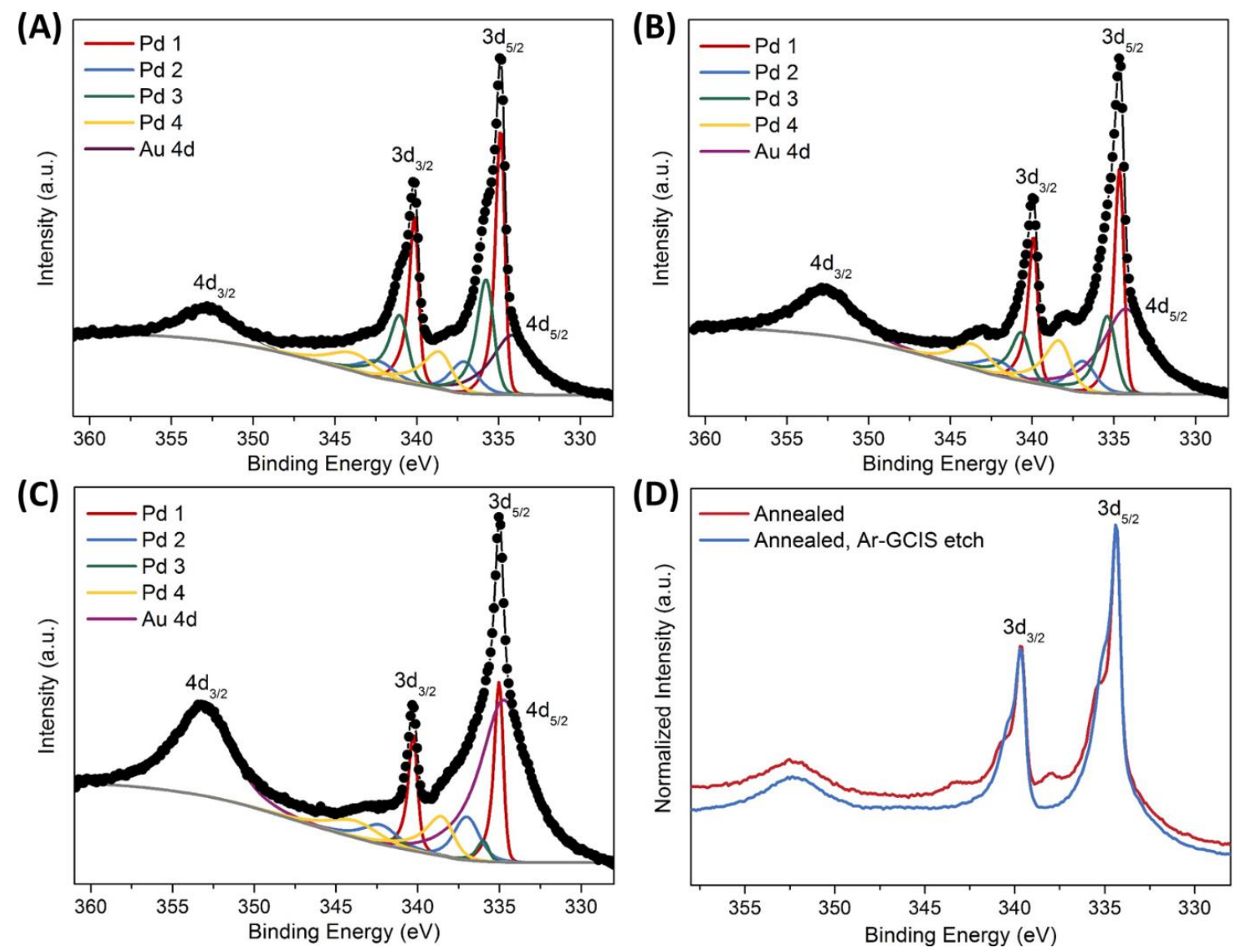

Figure 3 Selected, representative high-resolution XPS spectra of the Pd $3 d$ spectral region for AuPd alloyed nanoparticles with elemental ratios A) 1:3, B) 2:2 and C) 3:1. D) Normalized XPS Pd 3d spectra for alloyed nanoparticles before and after a short (10s) etch with an Ar gas cluster ion source (Ar-GCIS).

The $P d 3 d$ doublets at higher binding energies, relative to $P d(0)$, correspond to various higher oxidation states of Pd. We have assigned the Pd2 component, at approximately $335.6 \mathrm{eV}$, as Pd coordinated to 
oxygen through carbonyl, carboxyl and hydroxyl groups present in adventitious carbon or oxygen spillover from the $\mathrm{TiO}_{2}$ support (resulting from strong metal-support interaction). ${ }^{44}$ The $\mathrm{Pd} 3$ component at $337 \mathrm{eV}$ corresponds to $\mathrm{PdO}$ and the $\mathrm{Pd} 4$ doublet, with the $3 d_{5 / 2}$ peak at around 338.4 $\mathrm{eV}$, we assign to $\mathrm{PdO}_{2} \cdot{ }^{45}$ While bulk $\mathrm{PdO}_{2}$ is unstable, and XPS assignment using validated data is limited, it has been assigned in a range of nanoparticulate systems. ${ }^{44,46-49}$ The contribution of oxide species is significantly reduced by a short (10 s) argon gas cluster ion etch (Figure 3D), indicating it is highly localized at the nanoparticle surface. Interestingly, the relative fraction of the Pd4 component decreased from 29 to $8 \%$ as the alloy Pd content decreased from $75-25 \%$ (Figure $3 \mathrm{~A}-\mathrm{C}$ ), indicating $\mathrm{PdO}_{2}$ is less prevalent when neighboring more Au atoms.

The negative shift in binding energy of both $\mathrm{Au} 4 f$ and $\mathrm{Pd} 3 d$ electrons upon alloying is ascribed to the orbital hybridization of $\mathrm{Au}$ and $\mathrm{Pd}$ atoms. In brief, the loss and gain of respective $s p$ and $d$ electrons (i.e. negative charge) in Au corresponds to analogous positive charge loss and gain in Pd. This intraatomic charge redistribution of both metals, rather than the interatomic electron transfer from $\mathrm{Pd}$ to more electronegative $\mathrm{Au}$, is responsible for the widely observed composition-dependent catalysis of AuPd alloys.

The X-ray absorption near edge structure (XANES) spectra, measured over the $\mathrm{Au}_{\mathrm{L}}$-edge and Pd Kedge at the Australian Synchrotron, ${ }^{42}$ revealed only metallic $\mathrm{Au}$ and $\mathrm{Pd}$ in all alloyed samples. The $\mathrm{Au}$ $\mathrm{L}_{3}$-edge white line intensity for Au nanoparticles was lower relative to that of a gold foil, due to lower hybridisation of $5 d$ and $6 s p$ orbitals in nanoscaled structures (Figure S8), and reduced further still in all alloyed samples. Increasing the Pd concentration in alloyed particles decreased the $\mathrm{Au}_{\mathrm{L}} \mathrm{L}_{3}$-edge white line intensity (Figure S9) due to enhanced filling of the Au $5 d$ band by Pd, i.e. the Au $d$-hole count reduces with increasing Pd content. ${ }^{50-51}$ The Pd K-edge spectra showed metallic-like Pd in all alloy compositions (Figure $\mathbf{S 1 0 , 1 1 ) , ~ w i t h ~ t h e ~ s l i g h t ~ i n c r e a s e s ~ i n ~ e n e r g y ~ o f ~ t h e ~} 1 s \rightarrow 5 p$ and $1 s \rightarrow 4 f$ transitions corresponding to changes in both lattice spacing and $d$-band filling due to dilution of $\mathrm{Pd}$ in the $\mathrm{Au}$ matrix. ${ }^{51-52}$ Furthermore, the use of different substrates (Al mirror, $\mathrm{SiO}_{2}$ spacer, $\mathrm{TiO}_{2}$ deposited via atomic layer deposition, absence of mirror layer, Figure S8B, S10B) did not appreciably change the XANES spectra for either Au or Pd.

The matching coordination numbers (CN) of Au and Pd across all samples (Table 1), determined using extended X-ray absorption fine structure (EXAFS, for spectra and fitting see Figures S12-14 and discussion in Supporting Information), suggest that nanoparticles contained homogeneous distributions of atoms throughout. Furthermore, the metal bond lengths demonstrated that the lattice contracts similarly for $\mathrm{Au}-\mathrm{Au}, \mathrm{Pd}-\mathrm{Pd}$, and $\mathrm{Au}-\mathrm{Pd}$ bonds as the $\mathrm{Au}: \mathrm{Pd}$ ratio decreased, also consistent with a homogeneous alloy (Table 1). The magnitude and sign of the Cowley short-range order parameters corroborated this interpretation (Table 1), describing an environment where atoms of the same metal are unlikely to cluster. ${ }^{41}$ Core-shell Au@Pd nanoparticles (Figure S15) resulted in a Cowley parameter similar to thin $\mathrm{Au}$ and Pd films before annealing (Table S1). Furthermore, neither changing the Au:Pd ratio (Table $\mathbf{1}$ ) nor any alternative substrate (Table S1) greatly differed the Cowley parameter, confirming that the fabrication of alloyed nanoparticle via physical vapour deposition was robust and reproducible.

Table 1. Composition and electronic structure of alloyed AuPd nanoparticles.

\begin{tabular}{|c|c|c|c|}
\hline Au:Pd deposited thickness (nm) & $\underline{1: 3}$ & $\underline{2: 2}$ & $\underline{3: 1}$ \\
\hline \multicolumn{4}{|l|}{ Inductively coupled plasma mass spectrometry } \\
\hline$\chi_{\mathrm{Au}} / \chi_{\mathrm{Pd}}$ & 0.57 & 1.19 & 3.32 \\
\hline \multicolumn{4}{|l|}{ X-ray photoelectron spectroscopy ${ }^{a}$} \\
\hline$\chi_{\mathrm{Au}} / \chi_{\mathrm{Pd}}$ & $0.48(0)$ & $0.71(2)$ & $1.52(7)$ \\
\hline$\chi_{\mathrm{Au}} / \chi_{\mathrm{Pd}}[\mathrm{GI}-\mathrm{XPS}]$ & $0.54(1)$ & $0.89(0)$ & $-{ }^{b}$ \\
\hline Shift in $A u 4 f_{7 / 2}$ due to alloying (eV) & -0.5 & -0.7 & -0.2 \\
\hline Shift in $\mathrm{Pd} 3 \mathrm{~d}_{5 / 2}$ due to alloying (eV) & -0.2 & -0.4 & 0.0 \\
\hline Shift in Ti $2 p_{3 / 2}$ due to metal deposition (eV) & 0.5 & 0.1 & 0.4 \\
\hline
\end{tabular}




\begin{tabular}{|c|c|c|c|}
\hline \multicolumn{4}{|l|}{ Extended X-ray absorption fine structure ${ }^{a}$} \\
\hline$\chi_{\mathrm{Au}} / \chi_{\mathrm{Pd}}$ & $0.88(19)$ & $1.62(13)$ & $4.10(54)$ \\
\hline $\mathrm{N}_{\mathrm{Au}-\mathrm{Au}}$ & $3.4(6)$ & $5.8(5)$ & $8.0(5)$ \\
\hline$N_{P d-P d}$ & $5.1(6)$ & $3.7(5)$ & $1.3(8)$ \\
\hline $\mathrm{N}_{\mathrm{Au}-\mathrm{Pd}}$ & $6.1(4)$ & $4.4(3)$ & $2.2(3)$ \\
\hline $\mathrm{N}_{\text {Pd-Au }}$ & $5.4(12)$ & $7.1(7)$ & $9.1(18)$ \\
\hline $\mathrm{CN}_{\mathrm{Au}}$ & $9.5(14)$ & 10.2(9) & $10.2(18)$ \\
\hline $\mathrm{CN}_{\mathrm{Pd}}$ & $10.4(13)$ & $10.7(9)$ & $10.4(19)$ \\
\hline $\mathrm{R}_{\mathrm{Au}-\mathrm{Au}}(\AA)$ & $2.789(6)$ & $2.817(3)$ & $2.839(3)$ \\
\hline $\mathrm{R}_{\text {Pd-Pd }}(\AA)$ & $2.786(3)$ & $2.797(4)$ & $2.822(16)$ \\
\hline $\mathrm{R}_{\text {Au-Pd }}(\AA)$ & $2.786(3)$ & $2.804(3)$ & $2.824(4)$ \\
\hline Cowley short range parameter (Au) & -0.2 & -0.1 & -0.1 \\
\hline Cowley short range order parameter $(\mathrm{Pd})$ & -0.1 & -0.1 & -0.1 \\
\hline
\end{tabular}

\section{Optical properties and hot-carrier generation}

We determined the optical absorption (A) of the fabricated plasmonic near-perfect absorbers using experimental transmission $(T)$ and reflection ( $R$ ) spectra, according to the conservation of energy $\left(A=1-R-T\right.$, experimental spectra Figure S16). The bare $\mathrm{TiO}_{2}$ film exhibits no significant absorption bands across the visible spectrum, as expected for a high band-gap semiconductor (Figure 4A, cyan curve). Alloyed nanoparticles prepared on a semiconductor substrate reveal a broad but weak absorption across the visible spectrum with a small peak at $500 \mathrm{~nm}$, which we assign to the diminished plasmon resonance of $\mathrm{Au}$ in alloyed particles (green curve). The complete plasmonic near-perfect absorbers ( $\mathrm{Au} / \mathrm{TiO}_{2} / \mathrm{AuPd}$ of the type mirror/spacer/absorber) exhibited a distinct broadband absorption spanning 400-1000 nm with dual maxima at 470 and $570 \mathrm{~nm}$, the latter reaching a maximum absorption of $97 \%$ incident light (blue curve). Such high absorption arises from the drastically reduced reflectance of light (transmission remains negligible across the visible spectrum), which results from destructive interference among all possible light-scattering pathways in the structure. ${ }^{26}$ The critical coupling condition required to achieve high optical absorption is partly controlled by the thickness and refractive index of the $\mathrm{TiO}_{2}$ spacer layer ${ }^{30}$ Altering the Au:Pd ratio saw little change in band maxima although higher Pd content led to lower absorption intensities, $~ 80 \%$ in the 1:3 sample (Figure S17). We attribute this to the increasing imaginary part of the dielectric function as Pd content increases; as a lossy metal Pd dissipates more electronic energy than $\mathrm{Au}$, which is implicated in efficient charge-carrier generation. ${ }^{53}$

Fabricated plasmonic near-perfect absorbers exhibited anodic and cathodic photocurrents in both acidic $\left(100 \mathrm{mM} \mathrm{H}_{2} \mathrm{SO}_{4}\right)$ and alkaline $(500 \mathrm{mM} \mathrm{KOH})$ solutions without degrading the electrode. We attributed the large background photocurrent in acidic media (Figure S18) to capacitive behaviour induced by strong adsorption of $\mathrm{H}^{+}$to the electrode and therefore used alkaline conditions in all subsequent experiments. The observation of both photoanodic and cathodic behaviour (Figure S19) surprised us, as plasmonic near-perfect absorbers that contain monometallic Au nanoparticles on an n-type spacer act only as a photoanode; hot electrons, generated from excitation of the surface plasmon, are injected across the Schottky barrier into $\mathrm{TiO}_{2}$ to evolve oxygen. ${ }^{28,54}$ We instead assign the photocathodic mechanism to the direct reduction of adsorbed water molecules by hot electrons at the nanoparticle/solvent interface, evolving hydrogen via the two-step Volmer-Heyrovsky mechanism (Figure 4B). ${ }^{55}$ This same mechanism is also invoked in photocathodes comprised of monometallic Au nanoparticles deposited on a p-type $\mathrm{NiO}_{x}$ spacer. ${ }^{13}$ 
(A)

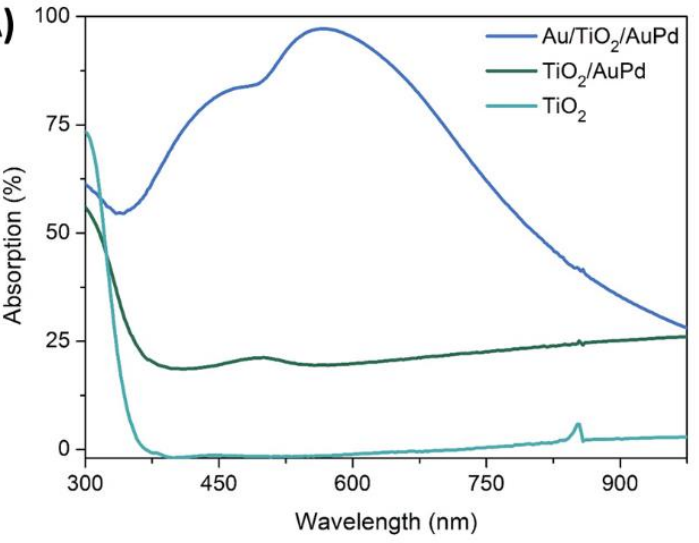

(C)

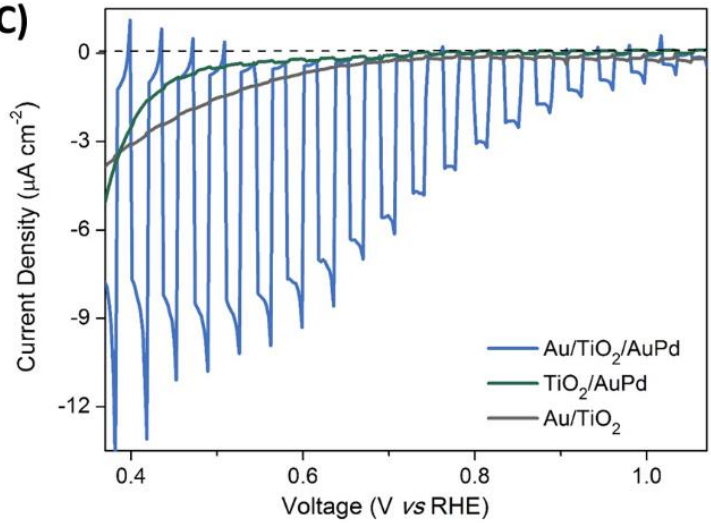

(B)
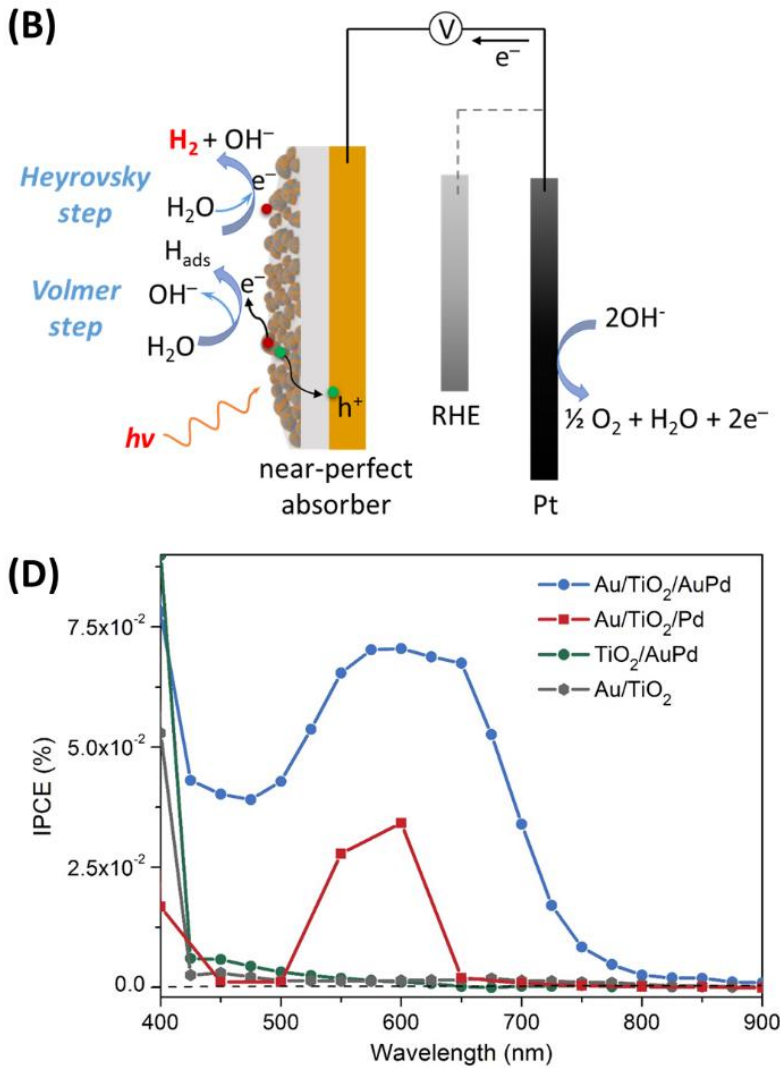

Figure 4. (a) Absorption spectra of fabricated plasmonic near-perfect absorber. (b) Schematic illustration of the photocurrent generation process in the PEC cell, $\mathrm{H}_{\text {ads }}$ is hydrogen atom adsorbed to the nanoparticle surface. (c) Linear sweep voltammogram under alkaline conditions (500 mM KOH) at $5 \mathrm{mV} \mathrm{s}^{-1}$ with chopped visible-light irradiation ( $3 \mathrm{~s},>495 \mathrm{~nm}$ ). (d) Action spectra using monochromatic light at $700 \mathrm{mV} v s \mathrm{RHE}$.

We assessed the photoresponse of plasmonic near-perfect absorbers via linear sweep voltammetry with chopped visible-light excitation, using a long-pass filter $(>495 \mathrm{~nm})$ to remove high-energy photons (Figure 4C). In the absence of a filter, we observed mild photoanodic currents at $1.0 \mathrm{~V}$ vs the reversible hydrogen electrode (RHE), which we assigned to direct excitation of electron-hole pairs in $\mathrm{TiO}_{2}$ (Figure S20). With an onset potential of $1.0 \mathrm{~V} v \mathrm{RHE}, \mathrm{Au} / \mathrm{TiO}_{2} / \mathrm{AuPd}$ near-perfect absorbers displayed cathodic photocurrents across the potential sweep, achieving a current density $J_{\text {photo }}=8.3 \mu \mathrm{A} \mathrm{cm}{ }^{-2}$ at a fixed potential of $0.52 \mathrm{~V}$ vs RHE. In contrast, the control electrodes, $\mathrm{Au} / \mathrm{TiO}_{2}$ and $\mathrm{TiO}_{2} / \mathrm{AuPd}$, showed no photoresponse (Figure 4C). Plasmonic near-perfect absorbers containing alloyed nanoparticles also exhibited significantly greater current densities than monometallic analogues, $J_{\text {photo }}=1.1$ and $0.6 \mu \mathrm{A} \mathrm{cm}^{-2}$ at $0.5 \mathrm{~V}$ vs RHE for $\mathrm{Au} / \mathrm{TiO}_{2} / \mathrm{Au}$ and $\mathrm{Au} / \mathrm{TiO}_{2} / \mathrm{Pd}$, respectively (Figures S21). It is important to note that we observed considerable variance in the photoresponse of the studied plasmonic nearperfect absorbers, sometimes as great as $\pm 1 \mu \mathrm{A}$ between different sample batches (Figure S22). Given the reproducibility of our alloyed nanoparticles, this variability likely arises from $\mathrm{TiO}_{2}$ deposition via electron-beam evaporation, which is highly sensitive to vacuum-chamber conditions and is difficult to control. ${ }^{56}$ We also observed that the annealing conditions used to form nanoparticles also significantly altered the photoelectrochemical behaviour of $\mathrm{Au} / \mathrm{TiO}_{2}$ control substrates (further discussion in Supporting Information, Figures S23).

The measured action spectra demonstrate the incident photon-to-electron conversion efficiency (IPCE) of plasmonic near-perfect absorbers as a function of wavelength. All three components (mirror, spacer, and absorbing nanoparticles) were required for efficient water reduction, with an IPCE that closely mirrored the absorption spectrum of the plasmonic near-perfect absorber (Figure 4D, Figure S24). The 
maximum measured efficiency, $0.07 \%$, occurred at wavelengths between $600-700 \mathrm{~nm}$. In contrast, control samples containing either $\mathrm{Au} / \mathrm{TiO}_{2}$ or $\mathrm{TiO}_{2} / \mathrm{AuPd}$ showed virtually no photocatalytic activity until irradiation energy was large enough to directly excite the semiconductor (< $400 \mathrm{~nm}$ ). We were surprised by the minimal response from the $\mathrm{TiO}_{2} / \mathrm{AuPd}$ sample containing no mirror, as alloyed particles do absorb visible light (Figure $4 \mathbf{A}$ ), but this observation reinforces the significant enhancement that the near-perfect absorber architecture affords. Notably, we were able to measure an IPCE response without the addition of a sacrificial agent previously required for monometallic derivatives. ${ }^{28}$

Most importantly, the alloyed particles exhibited a greater conversion efficiency than the analogous $\mathrm{Au} / \mathrm{TiO}_{2} / \mathrm{Pd}$ that reached a maximum IPCE of $0.03 \%$ at $600 \mathrm{~nm}$ (Figure 4D). While not generally considered a plasmonic metal, we have shown that Pd nanoparticles incorporated in a near-perfect absorber are capable of localizing the energy of absorbed photons. ${ }^{30}$ These results demonstrate that alloying $\mathrm{Au}$ and $\mathrm{Pd}$ increases the optical response relative to monometallic Pd nanoparticles, thus increasing the conversion efficiency. Action spectra for water reduction could not be measured with monometallic $\mathrm{Au} / \mathrm{TiO}_{2} / \mathrm{Au}$ substrates as they are unable to engage in this mechanistic pathway. $\mathrm{A}$ comparison of action spectra for the alternative photoanodic reaction (evolving oxygen) revealed that alloying had a negative impact on catalytic activity relative to plasmonic near-perfect absorbers that contained Au nanoparticles (Figure S25). The lossy Pd metal dampens the plasmonic properties of Au, therefore reducing hot electron formation and subsequent injection into the $\mathrm{TiO}_{2}$ layer.

In addition to the improved optical response afforded by both the near-perfect absorber and alloying Au with Pd, strong binding of water molecules to the catalyst surface is critical for ensuring efficient hydrogen evolution under alkaline conditions. ${ }^{55,57-58}$ Adsorption of molecules at a heterogenous transition metal surface is understood by $d$-band theory; stronger adsorption occurs when the $d$-band centre ( $\varepsilon_{d}$ is defined as the central position of the metal $d$-orbitals) is close to the metal's Fermi level. ${ }^{59}$ Hybridization of Au $5 d_{5 / 2}$ and Pd $4 d$ bands, as shown by XPS, shifts $\varepsilon_{d}$ closer to the Fermi level (Figure 5, step (i)) and increases the binding affinity of water. A similar increase in adsorption strength compared to monometallic analogues is observed for primary alcohols. ${ }^{50}$ The shift in $\varepsilon_{d}$ also strengthens the adsorption of hydrogen atoms $\left(H_{\text {ads }}\right)$, resulting in the hybridized bonding $(d-\sigma)$ and anti-bonding orbitals $\left(d-\sigma^{*}\right)$ between $\mathrm{H}_{\text {ads }}$ and the metal $d$-band (Figure 5, step (ii)) that provide a sufficient driving force for hot electron injection (step (iii)) and subsequent desorption of the $\mathrm{H}_{2}$ product. ${ }^{59-60}$

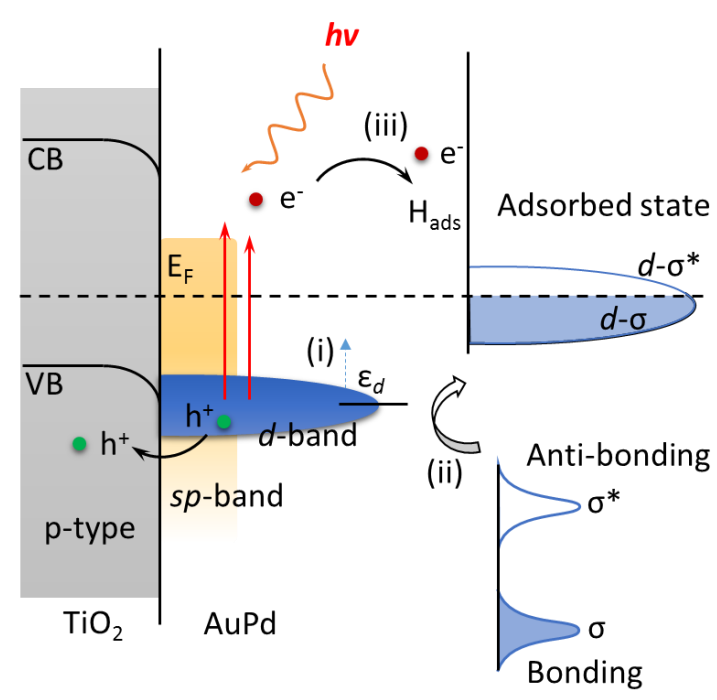

Figure 5. Representative energy diagram of fabricated near-perfect absorber and the orbital hybridization that occurs when $\mathrm{H}_{\text {ads }}$ binds to the catalyst surface. 


\section{Conclusions}

In summary, we outline a robust and reproducible protocol for preparing alloyed nanoparticles using lithographic-free physical vapour deposition. The extensive characterisation of AuPd nanoparticles prepared via this protocol revealed uniform distribution of both metals across many individual particles. The prepared nanoparticles were incorporated into the absorbing layer of a plasmonic nearperfect absorber that demonstrated up to $97 \%$ absorption of incident light. The fabricated plasmonic near-perfect absorbers demonstrated a strong photocathodic response, opposite to the anodic behaviour observed in plasmonic Au photoelectrodes on a n-type spacer. The AuPd alloy exhibited an increased optical response (in measured current density and IPCE) compared to monometallic palladium nanoparticles for photoelectrochemical hydrogen evolution under alkaline conditions. We attribute the overall improvement in photocurrent to the combined photon-to-energy conversion of $\mathrm{Au}$ (via plasmonic transitions) and $\mathrm{Pd}$ (via interband transitions) along with the improved surface adsorption of both $\mathrm{H}_{2} \mathrm{O}$ and $\mathrm{H}_{\text {ads }}$ that the catalytic Pd affords. Incorporating alloyed particles into an electromagnetic absorber further enhances the efficiency of hot-electron transfer and these materials represent a promising step towards photoelectrochemical catalysts that operate under real-world conditions without the need for chemical additives.

\section{Experimental Section}

\section{Plasmonic near-perfect absorber fabrication}

All films were deposited in a Nanochrome II electron beam evaporation system (IntIvac, Colorado, USA) using a $10 \mathrm{kV}$ power supply in a Class 10,000 cleanroom. First, a thin layer of $\operatorname{Cr}(5 \mathrm{~nm}, 0.5 \AA \mathrm{A} / \mathrm{s})$ was deposited as an adhesion layer onto cleanroom-grade glass slides (SCHOTT, Nexterion ${ }^{\circledR}$ B). Sequential deposition of a Au mirror layer $(100 \mathrm{~nm}, 1.0 \AA \mathrm{A} / \mathrm{s}), \mathrm{TiO}_{2}$ spacer layer $(30 \mathrm{~nm}, 0.5 \AA \mathrm{A} / \mathrm{s})$, and thin layers of $\mathrm{Au}$ and $\mathrm{Pd}(2 \mathrm{~nm}$ each, $0.1-0.4 \AA / \mathrm{s})$ was performed without exposing substrates to air. Coalescence of alloyed nanoparticles was achieved via annealing in a tube furnace at $500{ }^{\circ} \mathrm{C}$ for 30 min under Ar.

\section{Inductively coupled plasma mass spectrometry}

Thin films of $\mathrm{Au}$ and $\mathrm{Pd}$ deposited on glass slides were digested in aqua regia $(4 \mathrm{~mL})$ heated to $100{ }^{\circ} \mathrm{C}$ for $3 \mathrm{~h}$. After cooling, solutions were diluted to $50 \mathrm{~mL}$ using water. Analysis was performed on 7700 Series ICP-MS (Agilent, Mulgrave, Australia). This solution was analysed with appropriate standards using the ICP-MS.

\section{Microscopy}

Scanning electron microscope images of alloyed nanoparticles were obtained using a Merlin fieldemission scanning electron microscope (Zeiss, North Ryde, Australia) equipped with an In lens detector, operated at $3 \mathrm{kV}$. Samples were mounted on an aluminum stub with double-sided conductive carbon tape and imaged in secondary electron mode to highlight topographical features.

To prepare TEM samples the AuPd NPA substrate was gently scratched and detached particles suspended in ethanol. The suspended particles were transferred to a Cu grid and the solvent allowed to fully evaporate before imaging.

Transmission electron microscopy (TEM), scanning transmission electron microscopy (STEM) and selected area electron diffraction (SAED) were performed on a JEM2100F field-emission gun TEM (JEOL, Tokyo, Japan) operated at $200 \mathrm{kV}$ equipped with an Ultrascan1000 CCD (Gatan, Pleasanton, USA). STEM and energy dispersive X-ray (EDX) spectroscopy were carried out on a dual-aberrationcorrected Titan ${ }^{3}$ 80-300 TEM (FEI, Hillsboro, USA) operated at $300 \mathrm{kV}$ equipped with a Bruker $60 \mathrm{~mm}^{2}$ windowless SDD X-ray detector and analyser system. STEM imaging used both high-angle annular dark field and bright field image modes. The HAADF inner collection semi-angle was $>50 \mathrm{mrad}$, ensuring contrast was dominated by atomic number (Z). 


\section{X-Ray Diffraction}

A SmartLab X-ray diffractometer (Rigaku, Tokyo, Japan) equipped with a rotating anode CuKa source (45 kV, $200 \mathrm{~mA}$ ) and HyPix-3000 detector (Rigaku, Tokyo, Japan) was employed to obtain grazing incidence X-ray diffractograms. The diffractometer was configured with $0.35-0.55 \mathrm{~mm}$ incidence slits and a beam limiting mask of $5 \mathrm{~mm}$. Data was collected over the $2 \theta$ range $10-115^{\circ}$ with a step size of $0.02^{\circ}$ and a scan rate of $1^{\circ}$ per minute. Data was collected for each sample with $\omega$ fixed at $0.35^{\circ}, 0.45^{\circ}$ and $0.55^{\circ}$. The incidence slit, beam limiting mask and $\omega$ setting combine to maintain a beam footprint of $\sim 18 \mathrm{~mm} \sim 6 \mathrm{~mm}$, and the sample was also sat atop a Si zero background plate to ensure no contribution from the sample stage.

Analyses were performed on the collected XRD data using the EVA ${ }^{\mathrm{TM}}$ v5 software package (Bruker, Billerica, USA). Crystalline phases were identified using the ICDD-JCPDS powder diffraction database. Pawley analyses were performed on the data using TOPAS ${ }^{\mathrm{TM}}$ v6 (Bruker, Billerica, USA) for lattice parameter calculations. Background signal was described using a combination of Chebyshev polynomial linear interpolation function and $1 / x$ function. Cell parameters, vertical sample displacement, peak full width at half maximum, and peak scale factors were all refined. Error ranges were calculated on the basis of three estimated standard deviations as calculated using TOPAS $^{\mathrm{TM}}$.

\section{X-Ray Photoelectron Spectroscopy}

X-ray photoelectron spectroscopy (XPS) analysis was performed using an AXIS Nova and AXIS Ultra DLD spectrometers (Kratos Analytical Inc., Manchester, UK) using our standard protocol detailed elsewhere. ${ }^{61}$ The following parameters were employed during analysis: X-ray source and power monochromated Al K $\mathrm{K}_{\alpha}$ source at $180 \mathrm{~W}$; system pressure - between $10^{-9}$ and $10^{-8} \mathrm{mbar}$; pass energy $160 \mathrm{eV}$ (survey) and $20 \mathrm{eV}$ or $40 \mathrm{eV}$ (high resolution); step size $-0.5 \mathrm{eV}$ (survey) and $0.1 \mathrm{eV}$ (high resolution); emission angle $-0^{\circ}$ as measured from the surface normal; charge neutraliser - on.

In-situ etching was conducted using an Ar Gas Cluster Ion Source (GCIS; Kratos Analytical Inc. Minibeam 6) operated at a cluster size of $\mathrm{Ar}_{500+}$ with an impact energy of $10 \mathrm{keV}$, equating to a partition energy of $20 \mathrm{eV}$ per atom. For the ion beam a raster size of $1.5 \times 1.5 \mathrm{~mm}^{2}$ was employed. A stable beam current was confirmed prior to performing the depth profile experiment by measuring the sample current on the earthed sample platen (between 10 and $20 \mathrm{nA}$ ). The sample was etched once for $10 \mathrm{~s}$.

Data processing was performed using CasaXPS processing software version 2.3.15 (Casa Software Ltd., Teignmouth, UK). The atomic concentrations of the detected elements were calculated using integral peak intensities and the sensitivity factors supplied by the manufacturer. Binding energies were referenced to the Fermi edge measured by XPS at $0 \mathrm{eV}$. Protocol employed for peak fitting of spectra is detailed in the SI.

\section{X-ray Absorption Spectroscopy}

Au $\mathrm{L}_{3}$-edge and Pd K-edge XAS spectra were recorded on the multipole 1.9T wiggler XAS beam-line 12 ID in operational mode 1 and 2 at the Australian Synchrotron. The beam energy was $3.0 \mathrm{GeV}$. Samples were prepared on cleanroom glass slides (BOROFLOAT ${ }^{\circledR} 33$, NEXTERION ${ }^{\circledR}$ Glass B) via physical vapor deposition, and cooled to $10 \mathrm{~K}$ using a liquid helium cryostat. Absorption spectra of reference foils was measured in transmission, while sample spectra were measured in fluorescence mode up to $k=16 \AA^{-}$ ${ }^{1}$ post-edge.

The EXAFS spectra were reduced and analysed using the IFEFFIT library and DEMETER software package. ${ }^{62} \chi(k)$ was Fourier-transformed from $k$ to $R$ space over the k-window 3-14 $\AA^{-1}$ and fit in Rspace between 1.6-3.25 $\AA$. To extract coordination numbers of Au and Pd as well as their bond lengths, only the first coordination shell was fitted. 
For all the samples measured, analysis was done by fitting theoretical FEFF6 signals to multi-edge data sets simultaneously. The single scattering monometallic paths ( $\mathrm{Au}-\mathrm{Au}$ and $\mathrm{Pd}-\mathrm{Pd}$ ) were calculated using FEFF calculations on their fcc bulk crystal structures. Heterometallic paths ( $\mathrm{Au}-\mathrm{Pd}$ and $\mathrm{Pd}-\mathrm{Au}$ ) were generated through replacing all the nearest neighbours with the opposite element in the coordinate list, i.e. for Au-Pd paths the Au FEFF input file was used with a Au core and all other lattice points occupied by a $\mathrm{Pd}$ atom. The amplitudes $\left(\mathrm{S}_{0}{ }^{2}\right)$ was fixed to 0.85 and 0.83 for $\mathrm{Au}$ and $\mathrm{Pd}$ respectively, and the $\Delta \mathrm{E}$ correction to the photoelectron energy was different for each edge measured. The bond lengths and mean squared bond length disorders for the Au-Pd bond were constrained between the measurements at the different edges as: $R_{\text {Au-Pd }}=R_{\text {Pd-Au }}$ and $\sigma_{\text {Au-Pd }}^{2}=\sigma_{\text {Pd-Au. }}^{2}$ The Au:Pd ratio was extracted through the relationship: $N_{A u-P d}=\frac{\chi_{P d}}{\chi_{A u}} N_{P d-A u}$. These constraints, along with fitting the number of nearest neighbours led to a total of 12 fit parameters, and the data in the fit window contained 23 independent points. Inclusion of any Pd-O scattering paths did not improve the fits and, in most instances, increased the reduced chi-square of the fit.

\section{Optical spectroscopy}

The diffuse and specular reflectance $(\mathrm{R})$ and transmittance $(\mathrm{T})$ spectra were measured using a Lambda 1050 spectrophotometer equipped with a $150 \mathrm{~mm}$ InGaAs integrating sphere (Perkin Elmer, Glen Waverly, Australia). With these two measurements, the absorbance (A) was calculated as $A=1-R-T$.

\section{Photoelectrochemistry}

Photoresponse measurements were carried out in a three-electrode cell using a PGSTAT2-4 potentiostat (Metrohm AutoLab, Utrecht, Netherlands). Fabricated plasmonic near-perfect absorbers served as the working electrode, combined with a reversible hydrogen reference electrode and $\mathrm{Pt}$ mesh counter electrode. Devices were illuminated $\left(0.64 \mathrm{~cm}^{2}\right.$ exposure area) with simulated light (AM 1.5G) from a UXL-302-O $300 \mathrm{~W}$ xenon gas discharge lamp (Ushio, Tokyo, Japan) using a $495 \mathrm{~nm}$ longpass filter unless otherwise specified, (Thorlabs, Newton, USA). Light intensity was calibrated to 100 $\mathrm{mW} \mathrm{cm}$ (in the absence of filter) prior to each experiment using a PM100D optical power and energy meter (Thorlabs, Newton, USA). Acidic ( $\left.100 \mathrm{mM} \mathrm{H}_{2} \mathrm{SO}_{4}\right)$ and basic $(500 \mathrm{mM} \mathrm{KOH})$ electrolyte solutions were used to study the photocatalytic behaviour of plasmonic near-perfect absorbers. All the electrolyte solutions were made using ultrapure water (MilliQ) and sparged with Ar for 15 min prior to measurement. Polarization curves for photoelectrodes were swept linearly from positive to negative at a scan rate of $5 \mathrm{mV} \mathrm{s}^{-1}$. The IPCE experiments were carried out using Oriel Cornerstone 130 1/8 m Monochromator (Newport, Irvine, USA) without altering individual wavelength intensities, which were calibrated using an optical power meter and calculated according to the following formula:

$$
I P C E=100 \times \frac{I\left(\mathrm{~A} / \mathrm{cm}^{2}\right)}{P\left(\mathrm{~W} / \mathrm{cm}^{2}\right)} \times \frac{1240}{\lambda(\mathrm{nm})}
$$




\section{AUTHOR INFORMATION}

\section{Corresponding Author}

Email: Anthony S. R. Chesman: anthony.chesman@csiro.au

Daniel E. Gómez: daniel.gomez@rmit.edu.au

\section{Present Addresses}

Q.X.: Research Initiative for Supra-Materials, Shinshu University, Nagano-shi, Nagano 380-8553, Japan

\section{Author Contributions}

Q.X., K.K. and C.K. prepared and characterised plasmonic near perfect absorber samples. C.K. led the XANES and EXAFS synchrotron measurements (with assistance from Q.X., K.K. and C.D.E.) and performed all data analysis. G.O.B. prepared core-shell nanoparticles for synchrotron experiments. K.K. and N.W.D. performed the photoelectrochemical measurements. Specialist characterisation using GI-XRD, HR-(S)TEM, EDX spectroscopy and XPS was performed by A.S., L.B. and C.D.E., respectively. The project was conceived and developed by Q.X., T.U.C., A.S.R.C. and D.E.G., including the discussion and analysis of all experimental results. T.C. prepared the manuscript with contributions and approval from all authors.

\section{Notes}

The authors declare no competing financial interest.

\section{ACKNOWLEDGMENT}

This work was performed in part at the Melbourne Centre for Nanofabrication (MCN) in the Victorian Node of the Australian National Fabrication Facility (ANFF). This research was undertaken on the Xray Absorption Spectroscopy beamline at the Australian Synchrotron, part of the Australian Nuclear Science and Technology Organisation (ANSTO).. Q.X, K.K. and C.K. were supported by OCE Fellowships from CSIRO. D.E.G. acknowledges the Australian Research Council (ARC) for support through a Future Fellowship (FT140100514). The authors acknowledge the use of instruments and scientific and technical assistance of the Monash Centre for Electron Microscopy, Monash University, the Victorian Node of Microscopy Australia. This research used equipment funded by Australian Research Council grant LE0454166.

\section{REFERENCES}

1. Linic, S.; Christopher, P.; Ingram, D. B., Plasmonic-metal nanostructures for efficient conversion of solar to chemical energy. Nat. Mater. 2011, 10 (12), 911-921.

2. Linic, S.; Aslam, U.; Boerigter, C.; Morabito, M., Photochemical transformations on plasmonic metal nanoparticles. Nat. Mater. 2015, 14 (6), 567-576.

3. Aslam, U.; Rao, V. G.; Chavez, S.; Linic, S., Catalytic conversion of solar to chemical energy on plasmonic metal nanostructures. Nat. Catal. 2018, 1 (9), 656-665.

4. $\quad$ Christopher, P.; Moskovits, M., Hot Charge Carrier Transmission from Plasmonic Nanostructures. Annu. Rev. Phys. Chem. 2017, 68 (1), 379-398.

5. Gellé, A.; Jin, T.; de la Garza, L.; Price, G. D.; Besteiro, L. V.; Moores, A., Applications of Plasmon-Enhanced Nanocatalysis to Organic Transformations. Chem. Rev. 2020, 120 (2), 986-1041. 
6. Xiao, Q.; Jaatinen, E.; Zhu, H., Direct Photocatalysis for Organic Synthesis by Using Plasmonic-Metal Nanoparticles Irradiated with Visible Light. Chem. Asian J. 2014, 9 (11), 3046-3064.

7. Tada, H., Overall water splitting and hydrogen peroxide synthesis by gold nanoparticle-based plasmonic photocatalysts. Nanoscale Adv. 2019, 1 (11), 4238-4245.

8. Clavero, C., Plasmon-induced hot-electron generation at nanoparticle/metal-oxide interfaces for photovoltaic and photocatalytic devices. Nat. Photonics 2014, 8 (2), 95-103.

9. Tung, R. T., The physics and chemistry of the Schottky barrier height. Appl. Phys. Rev. 2014, 1 (1), 011304.

10. White, T. P.; Catchpole, K. R., Plasmon-enhanced internal photoemission for photovoltaics: Theoretical efficiency limits. Appl. Phys. Lett. 2012, 101 (7), 073905.

11. Wu, K.; Chen, J.; McBride, J. R.; Lian, T., Efficient hot-electron transfer by a plasmon-induced interfacial charge-transfer transition. Science 2015, 349 (6248), 632.

12. Mascaretti, L.; Dutta, A.; Kment, Š.; Shalaev, V. M.; Boltasseva, A.; Zbořil, R.; Naldoni, A., Plasmon-Enhanced Photoelectrochemical Water Splitting for Efficient Renewable Energy Storage. Adv. Mater. 2019, 31 (31), 1805513.

13. Robatjazi, H.; Bahauddin, S. M.; Doiron, C.; Thomann, I., Direct Plasmon-Driven Photoelectrocatalysis. Nano Lett. 2015, 15 (9), 6155-6161.

14. Zheng, Z.; Tachikawa, T.; Majima, T., Plasmon-Enhanced Formic Acid Dehydrogenation Using Anisotropic Pd-Au Nanorods Studied at the Single-Particle Level. J. Am. Chem. Soc. 2015, 137 (2), 948-957.

15. Wang, F.; Li, C.; Chen, H.; Jiang, R.; Sun, L.-D.; Li, Q.; Wang, J.; Yu, J. C.; Yan, C.-H., Plasmonic Harvesting of Light Energy for Suzuki Coupling Reactions. J. Am. Chem. Soc. 2013, 135 (15), 55885601.

16. Wang, C.; Yin, H.; Chan, R.; Peng, S.; Dai, S.; Sun, S., One-Pot Synthesis of Oleylamine Coated AuAg Alloy NPs and Their Catalysis for CO Oxidation. Chem. Mater. 2009, 21 (3), 433-435.

17. Huang, X.; Li, Y.; Chen, Y.; Zhou, H.; Duan, X.; Huang, Y., Plasmonic and Catalytic AuPd Nanowheels for the Efficient Conversion of Light into Chemical Energy. Angew. Chem. Int. Ed. 2013, $52(23), 6063-6067$.

18. van der Hoeven, J. E. S.; Welling, T. A. J.; Silva, T. A. G.; van den Reijen, J. E.; La Fontaine, C.; Carrier, X.; Louis, C.; van Blaaderen, A.; de Jongh, P. E., In Situ Observation of Atomic Redistribution in Alloying Gold-Silver Nanorods. ACS Nano 2018, 12 (8), 8467-8476.

19. Hansgen, D. A.; Vlachos, D. G.; Chen, J. G., Using first principles to predict bimetallic catalysts for the ammonia decomposition reaction. Nat. Chem. 2010, 2 (6), 484-489.

20. Ham, H. C.; Hwang, G. S.; Han, J.; Nam, S. W.; Lim, T. H., Geometric Parameter Effects on Ensemble Contributions to Catalysis: $\mathrm{H}_{2} \mathrm{O}_{2}$ Formation from $\mathrm{H}_{2}$ and $\mathrm{O}_{2}$ on AuPd Alloys. A First Principles Study. J. Phys. Chem. C 2010, 114 (35), 14922-14928.

21. Groß, A., Reactivity of Bimetallic Systems Studied from First Principles. Top. Catal. 2006, 37 (1), 29-39.

22. Tedsree, K.; Li, T.; Jones, S.; Chan, C. W. A.; Yu, K. M. K.; Bagot, P. A. J.; Marquis, E. A.; Smith, G. D. W.; Tsang, S. C. E., Hydrogen production from formic acid decomposition at room temperature using a Ag-Pd core-shell nanocatalyst. Nat. Nanotechnol. 2011, 6 (5), 302-307.

23. Tedsree, K.; Chan, C. W. A.; Jones, S.; Cuan, Q.; Li, W.-K.; Gong, X.-Q.; Tsang, S. C. E., ${ }^{13}$ C NMR Guides Rational Design of Nanocatalysts via Chemisorption Evaluation in Liquid Phase. Science 2011, 332 (6026), 224.

24. Wang, Z.-L.; Yan, J.-M.; Ping, Y.; Wang, H.-L.; Zheng, W.-T.; Jiang, Q., An Efficient CoAuPd/C Catalyst for Hydrogen Generation from Formic Acid at Room Temperature. Angew. Chem. Int. Ed. 2013, 52 (16), 4406-4409.

25. Valenti, M.; Venugopal, A.; Tordera, D.; Jonsson, M. P.; Biskos, G.; Schmidt-Ott, A.; Smith, W. A., Hot Carrier Generation and Extraction of Plasmonic Alloy Nanoparticles. ACS Photonics 2017, 4 (5), 1146-1152. 
26. Ng, C.; Wesemann, L.; Panchenko, E.; Song, J.; Davis, T. J.; Roberts, A.; Gómez, D. E., Plasmonic Near-Complete Optical Absorption and Its Applications. Adv. Opt. Mater. 2019, 7 (14), 1801660.

27. Shi, X.; Ueno, K.; Oshikiri, T.; Sun, Q.; Sasaki, K.; Misawa, H., Enhanced water splitting under modal strong coupling conditions. Nat. Nanotechnol. 2018, 13 (10), 953-958.

28. Ng, C.; Cadusch, J. J.; Dligatch, S.; Roberts, A.; Davis, T. J.; Mulvaney, P.; Gómez, D. E., Hot Carrier Extraction with Plasmonic Broadband Absorbers. ACS Nano 2016, 10 (4), 4704-4711.

29. Xiao, Q.; Connell, T. U.; Cadusch, J. J.; Roberts, A.; Chesman, A. S. R.; Gómez, D. E., HotCarrier Organic Synthesis via the Near-Perfect Absorption of Light. ACS Catal. 2018, 8 (11), 1033110339.

30. Connell, T. U.; Bonin, G. O.; Easton, C. D.; Della Gaspera, E.; Chesman, A. S. R.; Davis, T. J.; Gómez, D. E., Directing Energy into a Subwavelength Nonresonant Metasurface across the Visible Spectrum. ACS Appl. Energy Mater. 2019, 2 (2), 1155-1161.

31. Shi, Q.; Connell, T. U.; Xiao, Q.; Chesman, A. S. R.; Cheng, W.; Roberts, A.; Davis, T. J.; Gómez, D. E., Plasmene Metasurface Absorbers: Electromagnetic Hot Spots and Hot Carriers. ACS Photonics 2019, 6 (2), 314-321.

32. Cortie, M. B.; McDonagh, A. M., Synthesis and Optical Properties of Hybrid and Alloy Plasmonic Nanoparticles. Chem. Rev. 2011, 111 (6), 3713-3735.

33. You, H.; Yang, S.; Ding, B.; Yang, H., Synthesis of colloidal metal and metal alloy nanoparticles for electrochemical energy applications. Chem. Soc. Rev. 2013, 42 (7), 2880-2904.

34. Antosiewicz, T. J.; Apell, S. P.; Zäch, M.; Zorić, I.; Langhammer, C., Oscillatory Optical Response of an Amorphous Two-Dimensional Array of Gold Nanoparticles. Phys. Rev. Lett. 2012, 109 (24), 247401.

35. Nugroho, F. A. A.; landolo, B.; Wagner, J. B.; Langhammer, C., Bottom-Up Nanofabrication of Supported Noble Metal Alloy Nanoparticle Arrays for Plasmonics. ACS Nano 2016, 10 (2), 2871-2879. 36. Wadell, C.; Nugroho, F. A. A.; Lidström, E.; landolo, B.; Wagner, J. B.; Langhammer, C., Hysteresis-Free Nanoplasmonic Pd-Au Alloy Hydrogen Sensors. Nano Lett. 2015, 15 (5), 3563-3570. 37. Bannenberg, L. J.; Nugroho, F. A. A.; Schreuders, H.; Norder, B.; Trinh, T. T.; Steinke, N.-J.; van Well, A. A.; Langhammer, C.; Dam, B., Direct Comparison of PdAu Alloy Thin Films and Nanoparticles upon Hydrogen Exposure. ACS Appl. Mater. Interfaces 2019, 11 (17), 15489-15497. 38. Kadkhodazadeh, S.; Nugroho, F. A. A.; Langhammer, C.; Beleggia, M.; Wagner, J. B., Optical Property-Composition Correlation in Noble Metal Alloy Nanoparticles Studied with EELS. ACS Photonics 2019, 6 (3), 779-786.

39. Darmadi, I.; Nugroho, F. A. A.; Kadkhodazadeh, S.; Wagner, J. B.; Langhammer, C., Rationally Designed PdAuCu Ternary Alloy Nanoparticles for Intrinsically Deactivation-Resistant Ultrafast Plasmonic Hydrogen Sensing. ACS Sens. 2019, 4 (5), 1424-1432.

40. Chen, P.-C.; Liu, G.; Zhou, Y.; Brown, K. A.; Chernyak, N.; Hedrick, J. L.; He, S.; Xie, Z.; Lin, Q.Y.; Dravid, V. P.; O'Neill-Slawecki, S. A.; Mirkin, C. A., Tip-Directed Synthesis of Multimetallic Nanoparticles. J. Am. Chem. Soc. 2015, 137 (28), 9167-9173.

41. Frenkel, A. I.; Wang, Q.; Sanchez, S. I.; Small, M. W.; Nuzzo, R. G., Short range order in bimetallic nanoalloys: An extended X-ray absorption fine structure study. J. Chem. Phys. 2013, 138 (6), 064202.

42. Frenkel, A. I., Applications of extended X-ray absorption fine-structure spectroscopy to studies of bimetallic nanoparticle catalysts. Chem. Soc. Rev. 2012, 41 (24), 8163-8178.

43. Militello, M. C.; Simko, S. J., Elemental Palladium by XPS. Surf. Sci. Spectra 1994, 3 (4), 387394.

44. Kim, K. S.; Gossmann, A. F.; Winograd, N., X-ray photoelectron spectroscopic studies of palladium oxides and the palladium-oxygen electrode. Anal. Chem. 1974, 46 (2), 197-200.

45. Militello, M. C.; Simko, S. J., Palladium Oxide (PdO) by XPS. Surf. Sci. Spectra 1994, 3 (4), 395401. 
46. Semagina, N.; Renken, A.; Laub, D.; Kiwi-Minsker, L., Synthesis of monodispersed palladium nanoparticles to study structure sensitivity of solvent-free selective hydrogenation of 2-methyl-3butyn-2-ol. J. Catal. 2007, 246 (2), 308-314.

47. Kibis, L. S.; Stadnichenko, A. I.; Koscheev, S. V.; Zaikovskii, V. I.; Boronin, A. I., Highly Oxidized Palladium Nanoparticles Comprising $\mathrm{Pd}^{4+}$ Species: Spectroscopic and Structural Aspects, Thermal Stability, and Reactivity. J. Phys. Chem. C 2012, 116 (36), 19342-19348.

48. Veziroglu, S.; Hwang, J.; Drewes, J.; Barg, I.; Shondo, J.; Strunskus, T.; Polonskyi, O.; Faupel, F.; Aktas, O. C., PdO nanoparticles decorated $\mathrm{TiO}_{2}$ film with enhanced photocatalytic and selfcleaning properties. Materials Today Chemistry 2020, 16, 100251.

49. Otto, K.; Haack, L. P.; deVries, J. E., Identification of two types of oxidized palladium on $Y^{-}$ alumina by X-ray photoelectron spectroscopy. Appl. Catal. B 1992, 1 (1), 1-12.

50. Zhu, X.; Guo, Q.; Sun, Y.; Chen, S.; Wang, J.-Q.; Wu, M.; Fu, W.; Tang, Y.; Duan, X.; Chen, D.; Wan, Y., Optimising surface $d$ charge of AuPd nanoalloy catalysts for enhanced catalytic activity. Nat. Commun. 2019, 10 (1), 1428.

51. Marx, S.; Baiker, A., Beneficial Interaction of Gold and Palladium in Bimetallic Catalysts for the Selective Oxidation of Benzyl Alcohol. J. Phys. Chem. C 2009, 113 (15), 6191-6201.

52. Xin, H.; Vojvodic, A.; Voss, J.; Nørskov, J. K.; Abild-Pedersen, F., Effects of $d$-band shape on the surface reactivity of transition-metal alloys. Phys. Rev. B 2014, 89 (11), 115114.

53. Xiao, Q.; Sarina, S.; Jaatinen, E.; Jia, J.; Arnold, D. P.; Liu, H.; Zhu, H., Efficient photocatalytic Suzuki cross-coupling reactions on Au-Pd alloy nanoparticles under visible light irradiation. Green Chem. 2014, 16 (9), 4272-4285.

54. Hu, S.; Shaner, M. R.; Beardslee, J. A.; Lichterman, M.; Brunschwig, B. S.; Lewis, N. S., Amorphous $\mathrm{TiO}_{2}$ coatings stabilize $\mathrm{Si}, \mathrm{GaAs}$, and $\mathrm{GaP}$ photoanodes for efficient water oxidation. Science 2014, 344 (6187), 1005.

55. Sheng, W.; Myint, M.; Chen, J. G.; Yan, Y., Correlating the hydrogen evolution reaction activity in alkaline electrolytes with the hydrogen binding energy on monometallic surfaces. Energy Environ. Sci. 2013, 6 (5), 1509-1512.

56. Jang, H. K.; Whangbo, S. W.; Choi, Y. K.; Chung, Y. D.; Jeong, K.; Whang, C. N.; Lee, Y. S.; Lee, H. S.; Choi, J. Y.; Kim, G. H.; Kim, T. K., Titanium oxide films on Si(100) deposited by e-beam evaporation. J. Vac. Sci. Technol. A 2000, 18 (6), 2932-2936.

57. Men, Y.; Li, P.; Zhou, J.; Cheng, G.; Chen, S.; Luo, W., Tailoring the Electronic Structure of $\mathrm{Co}_{2} \mathrm{P}$ by N Doping for Boosting Hydrogen Evolution Reaction at All pH Values. ACS Catal. 2019, 9 (4), 3744-3752.

58. Parsons, R., The rate of electrolytic hydrogen evolution and the heat of adsorption of hydrogen. Trans. Faraday Soc. 1958, 54 (0), 1053-1063.

59. Zheng, Y.; Jiao, Y.; Jaroniec, M.; Qiao, S. Z., Advancing the Electrochemistry of the HydrogenEvolution Reaction through Combining Experiment and Theory. Angew. Chem. Int. Ed. 2015, 54 (1), 52-65.

60. Santos, E.; Schmickler, W., d-Band Catalysis in Electrochemistry. ChemPhysChem 2006, 7 (11), 2282-2285.

61. Easton, C. D.; Kinnear, C.; McArthur, S. L.; Gengenbach, T. R., Practical guides for x-ray photoelectron spectroscopy: Analysis of polymers. J. Vac. Sci. Technol. A 2020, 38 (2), 023207.

62. Ravel, B.; Newville, M., ATHENA, ARTEMIS, HEPHAESTUS: data analysis for X-ray absorption spectroscopy using IFEFFIT. J. Synchrotron Rad. 2005, 12 (4), 537-541. 